\title{
Differential vascular growth in postpneumonectomy compensatory lung growth
}

\author{
Lucas G. Fernandez, MD, DSc, ${ }^{a}$ Timothy D. Le Cras, PhD, ${ }^{b}$ Mirta Ruiz, MD, ${ }^{c}$ David K. Glover, PhD, ${ }^{c}$ Irving L. Kron, MD, ${ }^{a}$
} and Victor E. Laubach, $\mathrm{PhD}^{\mathrm{a}}$

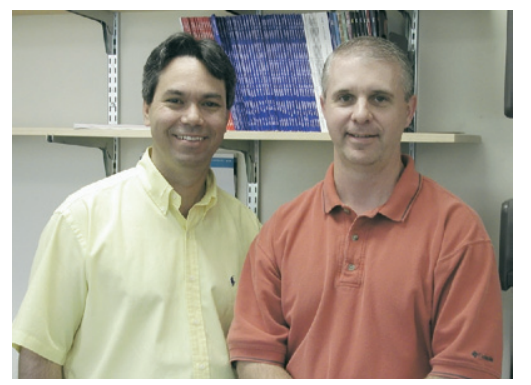

Drs Fernandez and Laubach (left to right)
From the Departments of Surgery ${ }^{\mathrm{a}}$ and Medicine, ${ }^{\mathrm{c}}$ University of Virginia Health System, Charlottesville, Va, and the Division of Pulmonary Biology, ${ }^{\mathrm{b}}$ Cincinnati Children's Hospital Medical Center, Cincinnati, Ohio.

Supported by Virginia Thoracic Society Career Investigator Award CI-52-N (V.E.L.), National Institutes of Health (NIH) HL67780 (V.E.L.), NIH HL72894 (T.D.L.C.), and the American Lung Association Career Investigator Award CI-31-N (T.D.L.C.)

Received for publication June 13, 2006; revisions received Aug 25, 2006; accepted for publication Sept 13, 2006.

Address for reprints: Victor E. Laubach, $\mathrm{PhD}$, University of Virginia Health System, Department of Surgery, PO Box 801359, Charlottesville, VA 22908 (E-mail: vel8n@ virginia.edu)

J Thorac Cardiovasc Surg 2007;133:309-16 $0022-5223 / \$ 32.00$

Copyright (C) 2007 by The American Association for Thoracic Surgery

doi:10.1016/j.jtcvs.2006.09.003
Objective: After pneumonectomy, compensatory growth occurs in the remaining lung. The vascular response during this growth and how individual lobes of the lung respond are not well understood. The aim of our study was to characterize vascular growth among individual lobes of the lung after pneumonectomy and determine whether changes in relative blood flow correlate with growth.

Methods: Rats underwent left pneumonectomy, and lobe weights and volumes of the right lung were measured 21 days later. Arterial growth was quantitated from arteriograms of each lobe after barium perfusion. Changes in relative blood flow were assessed by using radiolabeled microspheres. Expression of proliferating cell nuclear antigen was measured by means of Western blot analysis.

Results: After pneumonectomy, weight and volume indices of all lobes were significantly increased compared with those seen in sham control animals. Arterial growth occurred in all lobes after pneumonectomy, with the greatest increases occurring in the upper and middle lobes. In addition, a differential distribution of blood flow was observed where the upper and middle lobes contained the highest degree of relative flow. Pneumonectomy produced hyperplasic growth in all lobes, as indicated by significantly increased proliferating cell nuclear antigen expression. Proliferating cell nuclear antigen expression correlated with arterial growth in that increased and prolonged expression occurred in the upper lobe.

Conclusions: These results show that left pneumonectomy induces significant, nonuniform, compensatory growth in all lobes of the right lung. Arterial growth occurred in each lobe after pneumonectomy, but preferentially higher vascular growth and cell proliferation in the upper lobe positively correlated with higher relative blood flow in this lobe.

$\mathrm{T}$ he most important function of the lung is to provide $\mathrm{O}_{2} / \mathrm{CO}_{2}$ exchange. To maintain this balance after pneumonectomy, in experimental animals the remaining lung undergoes rapid compensatory growth, which restores lung weight, volume, DNA, and protein to control levels within 3 weeks. ${ }^{1-7}$ In addition, this response must coordinate both alveolar and vascular growth. A parallel increase in relative capillary volume and alveolar and capillary surface densities after bilobectomy in rats has been described. ${ }^{1}$ Our laboratory has recently shown that arterial growth occurs in the left lung after right lobectomy, which is accompanied by increased arterial area and length and is proportional to the amount of lung tissue (lobes) removed. ${ }^{8}$ However, the sequence of cellular events in compensatory lung growth remains poorly understood, and whether vascular growth is an initial event and precedes tissue growth or occurs simultaneously remains unknown. ${ }^{9}$

Immediately after left pneumonectomy, blood perfusion to the right lung increases $65 \%$ to $70 \%,{ }^{10}$ constituting a very powerful stimulus for potential vascular growth and proliferation. In addition, upregulation of angiogenic growth factors, 


\section{Abbreviations and Acronyms \\ PCNA $=$ proliferating cell nuclear antigen}

including vascular endothelial growth factor and its receptor, flk-1, has been reported in rats and mice after pneumonectomy. ${ }^{11,12}$ Relative changes in blood flow and lung expansion might be different among individual lobes of the remaining lung after pneumonectomy. An early study by Sekhon and Thurlbeck ${ }^{7}$ showed that compensatory growth is not equal in all 4 lobes of the right lung after left pneumonectomy; however, vascular growth was not addressed in this study. Therefore the aim of our study was to characterize the arterial growth response in compensatory growth among individual lobes of the right lung after left pneumonectomy and to determine whether changes in relative distribution of blood flow correlate with the arterial growth and proliferative response in the lung.

\section{Methods}

\section{Animals}

Adult male Sprague-Dawley rats weighing 300 to $350 \mathrm{~g}$ were used for all experiments (Charles River, Wilmington, Mass). Facilities for animal care are accredited by the American Association for Accreditation of Laboratory Animal Care. All procedures and protocols were approved by the Institutional Animal Care and Use Committees at the University of Virginia Health System, Charlottesville, Virginia, and at Children's Hospital Medical Center, Cincinnati, Ohio.

\section{Surgical Procedure}

The operative technique for left pneumonectomy was performed as previously described. ${ }^{13,14}$ Surgical intervention was carried out after achievement of halothane anesthesia administered through a nose cone. Animals in the pneumonectomy group underwent a posterolateral thoracotomy, and the left lung was freed from the inferior pulmonary ligament. The lung was then delivered into the surgical wound, the hilum was tied with a 3-0 silk ligature, and the lung was excised. The chest was closed with 3-0 nylon sutures, and the skin was closed with surgical staples. Animals in the sham group underwent a similar procedure, except the hilum was not tied, and the lung was not removed. Animals were allowed to recover from anesthesia and received postoperative analgesia in the form of buprenorphine hydrochloride $(0.03 \mathrm{mg} / \mathrm{kg}$ administered subcutaneously). The animals were allowed to feed ad libitum and were maintained in a controlled environment of normal temperature, humidity, and light cycle.

This study used a total of 65 rats, which were used in 4 separate sets of pneumonectomy and sham operations and summarized as follows: (1) one set of animals ( 25 total) underwent harvest at days 0,7 , and 21 ( $\mathrm{n}=5$ per group) to measure lung weight index (day 21 only) and for collection of fresh tissue for Western blot analysis; (2) a second set of animals (12 total) underwent harvest at day 21 ( $\mathrm{n}=6$ per group) to measure lung volume indices; (3) a third set of animals (12 total) underwent harvest at day $21(\mathrm{n}=6$ per group) to measure vascular area and arterial lengths through barium infusion and arteriograms; and (4) a fourth set of animals (16 total) was used to measure relative blood flow by using microspheres. These are described in detail below.

\section{Lung Harvest and Lung Weight Index}

Animals in the pneumonectomy and sham groups were killed on day 21 after the operation ( $n=5$ per group). Rats were anesthetized with a combination of ketamine and xylazine injected intraperitoneally, weighed, and intubated through a tracheotomy, and exposure of the organs was obtained by means of a bilateral anterior sternothoracotomy. The animals were rapidly exsanguinated by using renal vein division, the right lung was removed, and each lobe was carefully dissected, blotted dry, weighed, and then frozen for Western blot analysis. In addition, individual lobes from rats not undergoing operations and a separate set of sham and pneumonectomy animals were harvested at 7 days after the operation ( $\mathrm{n}=5$ per group), snap-frozen in liquid nitrogen, and stored at $-80^{\circ} \mathrm{C}$ for Western blot analysis only.

\section{Lung Volume Index}

A separate set of rats undergoing pneumonectomy and sham operations were killed at day 21 after the operation $(n=6$ per group) to obtain lung and lobe volumes. The lungs were removed and received intratracheal instillation of $4 \%$ paraformaldehyde (Fisher Scientific, Fair Lawn, NJ) to a pressure of $20 \mathrm{~cm} \mathrm{H}_{2} \mathrm{O}$. After 1 hour, the trachea was tied under pressure, and the total lung volume was obtained by means of volume displacement, as described by Scherle. ${ }^{15}$ Each individual lobe was then ligated, and the volumes were obtained by means of volume displacement. The lobe volumes (in milliliters) and weights (in grams) were expressed as a ratio to the final body weights of the animals (in grams) to obtain the volume and weight indices.

\section{Arterial Area Index and Length Measurements}

A separate set of animals were used in which the pulmonary arterial area index was measured in lungs that were infused with barium sulfate, as previously described. ${ }^{8}$ Briefly, rats undergoing pneumonectomy and sham operations $(\mathrm{n}=6$ per group) were anesthetized at 21 days after the operation. A thoracotomy was performed, and heparin $(10 \mathrm{U})$ was injected into the right ventricle to prevent blood from clotting in the lungs. The lungs were gently inflated with air through a tracheotomy through a stainless-steel gavage needle. The lungs were inflated with the chest partially open. Blood was then flushed from the lungs with heparinized saline $(1 \mathrm{U} / \mathrm{mL})$ infused through a catheter placed into the main pulmonary artery. A barium sulfate-gelatin mixture at $70^{\circ} \mathrm{C}$ was then infused into the main pulmonary artery at a pressure of $74 \mathrm{~mm}$ $\mathrm{Hg}$ for at least 5 minutes. The main pulmonary artery was tied under pressure, and the lungs were fixed by means of tracheal instillation of $4 \%$ paraformaldehyde to a constant pressure of 20 $\mathrm{cm} \mathrm{H}_{2} \mathrm{O}$ for 48 hours. The barium-filled arterial structures in the lungs were imaged by using high-resolution radiography (MX-20; Faxitron X-ray, Wheeling, Ill) and high-resolution x-ray film (Microvision; AGFA, Greenville, SC). Radiographs for each lobe were scanned and imaged by using a flat-bed scanner with a transparency adapter, and quantitation of the arterial area (white area of the arteriogram) was performed with Imagequant (Amer- 
sham Biosciences, Piscataway, NJ). Each lobe was separated and scanned in identical orientations to permit accurate comparisons, and the arterial area was indexed to body weight. The lengths of the arterial branches were measured for the first branch off the upper lobe artery by using the measuring tool of Photoshop (Adobe, San Jose, Calif) calibrated to a $1-\mathrm{cm}$ line on the $4 \times$ arteriogram. Arterial measurements were indexed to body weight to control for variations caused by body size.

\section{Relative Blood-flow Distribution}

Two experiments were conducted with a total of 16 animals to determine the relative distribution of blood flow to the different lobes after pneumonectomy. In the first (acute) experiment a group of rats undergoing pneumonectomy $(\mathrm{n}=5)$ were injected 10 minutes after the operation with approximately 150,000 microspheres through the tail vein. Injections were performed in spontaneously breathing, slightly anesthetized rats (methoxyflurane). Animals were positioned on their left side, allowing maximum expansion for the right lobes. Microsphere injections were then repeated in the same animals at 3, 24, and 72 hours after the operation, resulting in a total of 4 injections per animal. A group of rats not undergoing operations $(n=6)$ received a single injection to serve as a control group. A total of 4 different $15-\mu \mathrm{m}$ radiolabeled microspheres were used (Ce-141, Sr-85, Nb-95, and Sc-46; Perkin Elmer Life Sciences, Boston, Mass), and each injection was comprised of a different radiolabeled microsphere to distinguish results from the various injection times. The second (chronic) experiment $(\mathrm{n}=5)$ was similar, except that the radiolabeled microsphere injections were initiated 3 hours after the operation and repeated at 10 and 21 days after the operation. On day 21, 5 minutes after the final injection, the animals were killed and weighed, and their lungs were removed. The lobes were then individually harvested and counted in a gamma-well scintillation counter (MINAXI 5550; Packard Instruments, Downers Grove, Ill). Tissue counts were corrected for background, decay, and isotope spillover, and the ratio between counts per minute in each lobe and the corresponding lobe weight (in grams) was used as an index to determine the relative distribution of blood flow between the lobes. The results for each lobe were expressed as the percentage of total blood flow in the right lung.

\section{Proliferating Cell Nuclear Antigen Expression}

Total cell protein $(100 \mu \mathrm{g})$ from lung samples was fractionated on a $12 \%$ sodium dodecylsulfate polyacrylamide gel and transferred to nitrocellulose membrane by using an electrophoretic transfer cell (BioRad, Hercules, Calif). The membrane was blocked in 50 $\mathrm{mmol} / \mathrm{L}$ Tris $\mathrm{HCl}(\mathrm{pH} 7.4), 150 \mathrm{mmol} / \mathrm{L} \mathrm{NaCl}$, and $0.1 \%$ Tween containing $5 \%$ milk and then incubated with a monoclonal antiproliferating cell nuclear antigen (anti-PCNA) antibody (\#610664, 1:5000; BD Biosciences, San Diego, Calif) for 2 hours at room temperature. After washing with $50 \mathrm{mmol} / \mathrm{L}$ Tris $\mathrm{HCl}(\mathrm{pH} \mathrm{7.4)}$, $150 \mathrm{mmol} / \mathrm{L} \mathrm{NaCl}$, and $0.1 \%$ Tween, the membrane was incubated for 1 hour with goat anti-mouse IgG antibody coupled to horseradish peroxidase (\#31430, 1:40,000; Pierce, Rockford, Ill) and washed as before. Protein bands were visualized by means of chemiluminescence (Supersignal, Pierce), and the autoradiograph was quantitated by means of computerized densitometry (Alpha Innotech Corp, San Leandro, Calif). The membranes were then stripped (Restore, Pierce), and a polyclonal anti-actin antibody (\#sc-1615, 1:300; Santa Cruz Biotechnology Inc, Santa Cruz, Calif), followed by a rabbit anti-goat $\mathrm{IgG}$ antibody coupled to horseradish peroxidase (\#31402, 1:20,000; Pierce), was used as a control for loading consistency. Preliminary tests showed that expression of actin did not differ between groups.

\section{Statistical Analysis}

Data are presented as means \pm standard error of the mean. Statistical analysis was performed with SPSS 13.0 for Windows software (SPSS Inc, Chicago, Ill). Comparisons were made with 1-way analysis of variance and the post-hoc Bonferroni test.

\section{Results}

\section{Lung Weight Index After Pneumonectomy}

The total right lung weights were measured in the pneumonectomy versus sham groups at 21 days after the operation. The pneumonectomy group had significantly higher total lung weight index compared with the sham group (3.20 \pm 0.87 vs $2.21 \pm 0.06, P<.01)$. Analysis of each of the 4 lobes of the right lung revealed that lobe weight index increased significantly for the upper, middle, lower, and cardiac lobes by $45 \%, 42 \%, 41 \%$, and $59 \%$, respectively $(P<.01$; Figure $1, A)$.

\section{Lung Volume Index After Pneumonectomy}

The right lung and lobe volume indices were measured at 21 days after the operation in the pneumonectomy versus sham groups. The right lungs of rats in the pneumonectomy group showed a significant increase in total lung volume index versus that seen in the sham group $(34.41$ \pm 0.92 vs $26.04 \pm 0.81, P<.01)$. The lobe volume indices of the upper, middle, lower, and cardiac lobes in the pneumonectomy group were significantly increased by $57 \%, 34 \%, 28 \%$, and $70 \%$, respectively $(P<.05$ versus the sham group; Figure 1, B).

\section{Arterial Area Index and Branch Lengths}

Vascular growth in each lobe was determined by the ratio of the arterial area of the lobe arteriogram to body weight (arterial area index). Arteries with an external diameter of approximately $30 \mu \mathrm{m}$ and larger contribute to this measurement. A representative arteriogram is shown in Figure 2, A. After 21 days, the arterial area index was significantly increased in the upper, middle, lower, and cardiac lobes of rats in the pneumonectomy group by $76 \%, 57 \%, 38 \%$, and $47 \%$, respectively $(P<.05)$ compared with that seen in the sham group (Figure 2, B). The upper lobe displayed a significantly higher percentage increase in arterial area index compared with the lower and cardiac lobes $(P<.05)$.

The lengths between the upper lobe artery and the first branch of the upper lobe (segment A) and between the first and second branch (segment B) were measured from the 

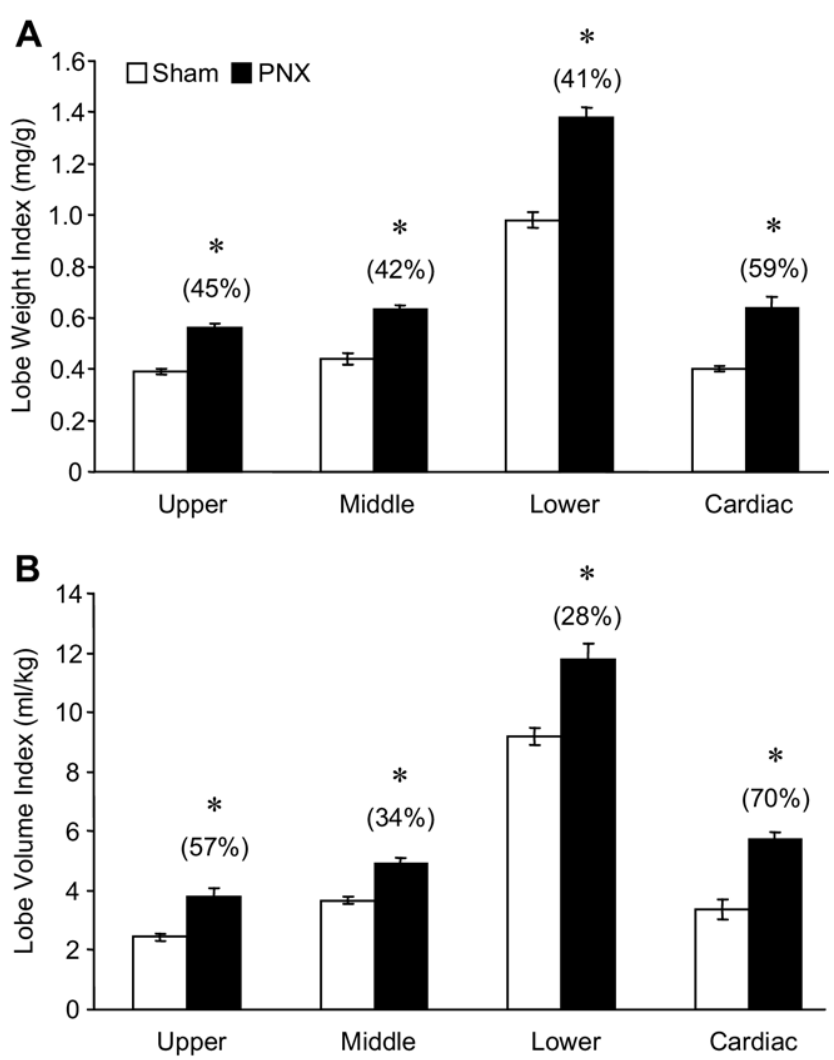

Figure 1. A, Changes in lobe weight index after pneumonectomy (PNX). Lobe weight index was significantly increased in all lobes 21 days after pneumonectomy versus that seen in the sham group $\left({ }^{*} P<.01\right)$. B, Changes in lobe volume index 21 days after pneumonectomy. Lobe volume index was significantly increased in all lobes after pneumonectomy versus that seen in the sham group $\left({ }^{*} \boldsymbol{P}<.05\right)$. Percentages of increase over the sham group are included in parentheses.

arteriograms of the upper lobe (Figure 3,A). The length of segment A increased 59\% after pneumonectomy compared with that seen in the sham control group $(P<.0001)$, whereas the length of segment B increased 33\% $(P=.0294$; Figure $3, B)$. The other lobes were not assessed because the 3-dimensional orientations of these lobes for arteriograms were not consistent enough to yield accurate measurements of branch lengths.

\section{Changes in Relative Blood Flow}

The relative distribution of blood flow among lobes was measured by means of serial injections of radiolabeled microspheres through the tail vein, as described in the "Methods" section.

In animals not undergoing operations ( 0 -minute time point), the upper lobe received $33 \%$ of the total right pulmonary blood flow, as measured by counts per minute/lobe
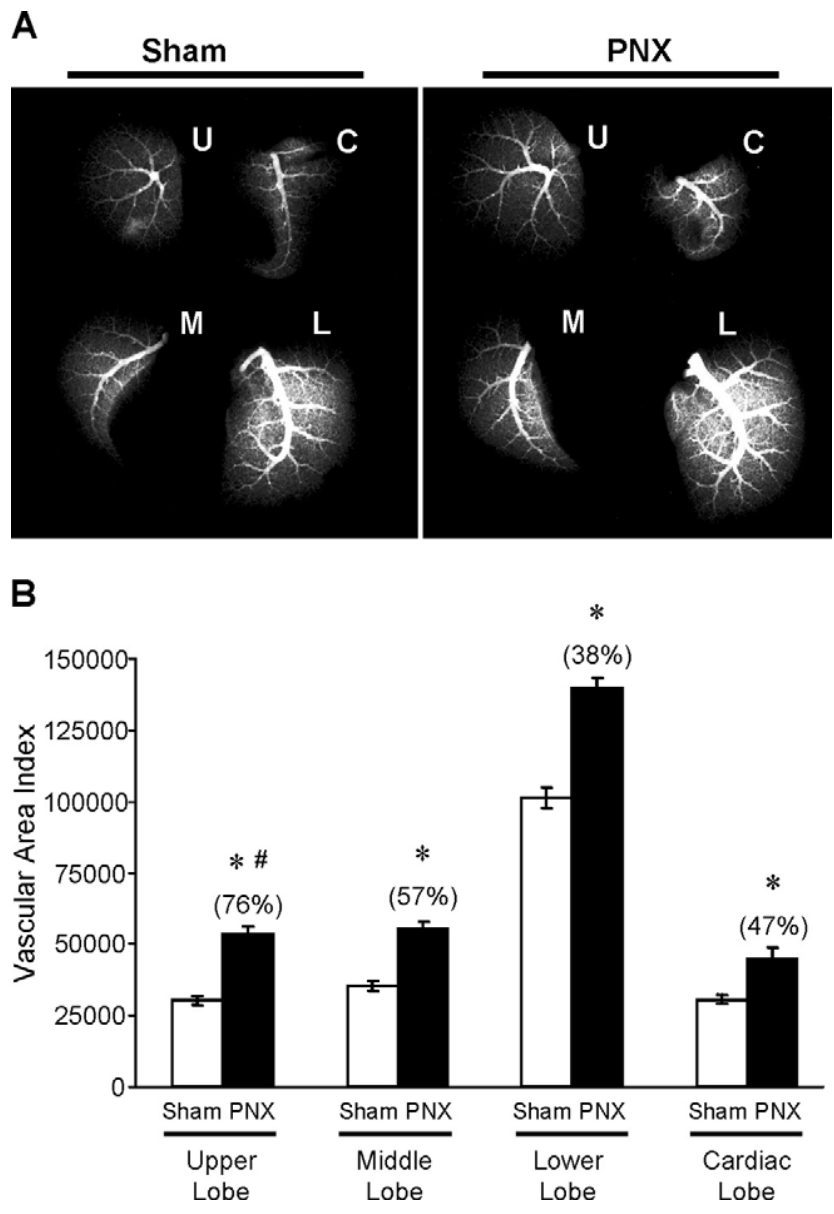

Figure 2. Changes in lobe arterial area index 21 days after pneumonectomy (PNX). A, Representative arteriograms of the 4 right lung lobes after sham and pneumonectomy operations. $U$, Upper lobe; $M$, middle lobe; $L$, lower lobe; $C$, cardiac lobe. B, Arterial area index, measured by means of arteriography, was significantly increased in all lobes after pneumonectomy versus sham operations $\left({ }^{*} P<.05\right)$, indicating an increase in overall vascular area (white areas) seen in each lobe of the pneumonectomy group. In the pneumonectomy group the upper lobe displayed a significant increase in arterial area index compared with the lower and cardiac lobes $(\# P<.05)$. Percentages of increase over the sham group are included in parentheses.

weight (in grams), and the lower lobe received the lowest portion of flow (13\%, Figure 4). The remaining lobes were very similar in relative flow: middle lobe, $26 \%$; cardiac lobe, $28 \%$. After left pneumonectomy, the entire blood flow is shifted to the right lung; however, the relative distribution of flow remained fairly constant among the lobes thereafter. The final distribution of relative blood flow among the 4 lobes at 21 days after pneumonectomy was as follows: upper lobe, 33\%; middle lobe, 29\%; cardiac lobe, $23 \%$; lower lobe, $15 \%$. 
A
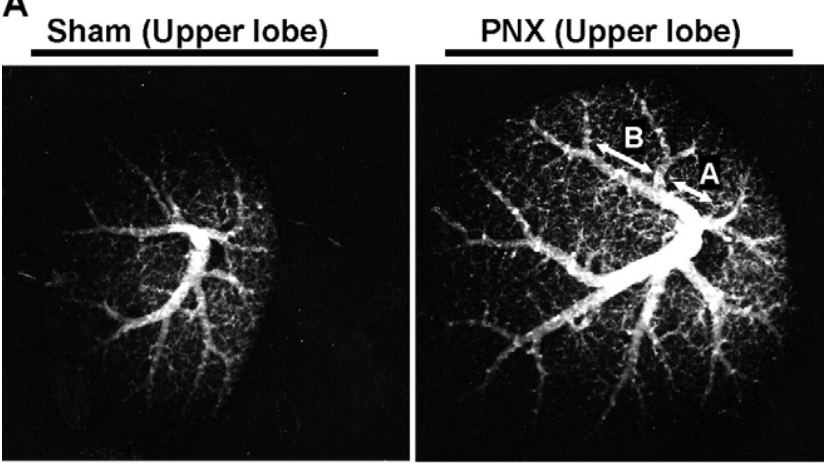

B

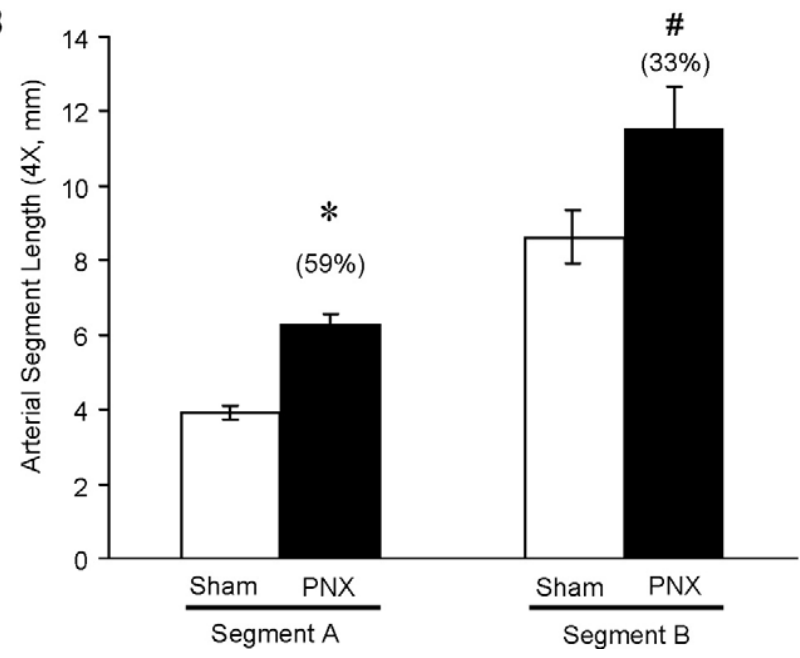

Figure 3. Changes in length of arterial branch segments in the upper lobe 21 days after pneumonectomy (PNX). A, An example of arteriograms of the right upper lobe after pneumonectomy and sham operations ( $4 \times$ magnification). The length between the upper lobe artery and the first branch (segment $A$ ) and the length between the first and second branches (segment $B$, arrows) were measured. $B$, Histogram showing that lengths of segment $A\left({ }^{*} P<\right.$ .0001 vs sham) and segment $B(\# P=.0294$ vs sham) are significantly increased after pneumonectomy. Percentages of increase over the sham group are included in parentheses.

\section{Right Ventricular Hypertrophy as an Index of}

\section{Pulmonary Hypertension}

There was no evidence of pulmonary hypertension in the pneumonectomy group because right ventricular hypertrophy, measured as the ratio of right ventricle/body weight, was not different between the pneumonectomy and sham groups 21 days after the operation. The right ventricle/body weight ratio was $0.056 \pm 0.03$ in the sham group $(\mathrm{n}=10)$ versus $0.060 \pm 0.05$ in the pneumonectomy group $(n=6)$.

\section{PCNA Protein Expression}

Cell proliferation in the lobes after pneumonectomy was assessed by measuring the expression of PCNA by means of
Western blot analysis. PCNA is a protein synthesized in the early G1 and S phase of the cell cycle, which serves as an excellent marker for proliferating cells. ${ }^{16}$ Figure 5, $A$, shows representative examples of PCNA Western blots. The relative PCNA/actin expression index was quantitated in each lobe at 7 and 21 days after the operation and compared with the corresponding lobes of rats not undergoing operations (day 0). Rats not undergoing operations were used as a control group because preliminary tests showed that expression of actin did not differ between these animals and rats undergoing sham operations at any time point after the operation (Figure 5, B). At day 7 after pneumonectomy, all lobes had a significant peak increase in PCNA expression (Figure 5,C). Here PCNA expression was increased by 10-fold (upper lobe), 3-fold (middle lobe), 6-fold (lower lobe), and 4-fold (cardiac lobe; $P<.01$ ). By day 21, PCNA expression was reduced in all lobes to levels similar to that seen in animals not undergoing operations, except in the upper lobe, in which expression remained significantly increased (4-fold, $P<.01$; Figure 5, $C$ ).

\section{Discussion}

Left pneumonectomy induces compensatory growth in all lobes of the right lung. The vascular response during this growth, as well as how individual lobes of the lung respond, is not well understood. Therefore the aim of this study was to characterize the arterial growth response during compensatory growth among individual lobes after left pneumonectomy and to determine whether changes in relative distribution of blood flow would correlate with the arterial growth and proliferative response in the lung.

In this study total lung weight and volume indices increased significantly after pneumonectomy, as expected. When individual lobes were examined, it was evident that compensatory growth occurred in all lobes. The upper and cardiac lobes had a higher increase in lobe weight index (45\% and 59\%, respectively) than the middle and lower lobes (42\% and $41 \%$, respectively) after pneumonectomy. In the context of lobe volume index, there was an even greater discrepancy observed in the upper and cardiac lobes. Here all 4 lobes displayed significant increases after pneumonectomy, with the upper and cardiac lobes showing a higher increase in lobe volume index $(57 \%$ and $70 \%$, respectively) than the middle and lower lobes (34\% and $28 \%$, respectively). Brody ${ }^{2}$ described an initial phase that accounted for increased lung weight in the first week after pneumonectomy, whereas lung volume did not increase until the second postoperative week. Sekhon and Thurlbeck $^{7}$ also showed that the upper and cardiac lobes had a greater increase in volume than the middle and lower lobes. Finally, Ravikumar and colleagues ${ }^{17}$ performed computed tomographic scans after left pneumonectomy in foxhounds and found unequal distribution of mechanical strain on the 


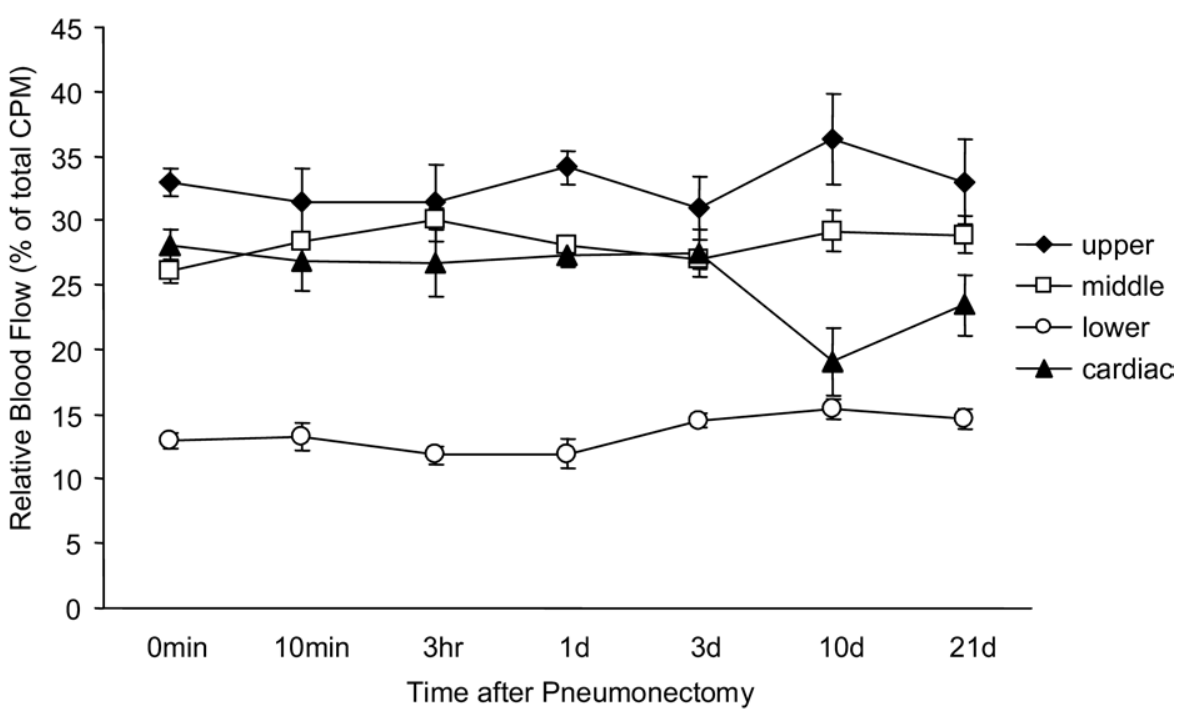

Figure 4. Changes in relative distribution of pulmonary blood flow after pneumonectomy. By using radiolabeled microspheres, relative blood flow (expressed as a percentage of total flow in the right lung) was determined in each lobe at the indicated times after pneumonectomy and compared with blood flow in animals not undergoing operations $(0$-minute time point). Relative blood flow was maintained immediately after pneumonectomy in each lobe $(10$-minute time point), with little change occurring thereafter. Relative blood flow in each lobe was not significantly different compared with that seen in animals not undergoing operations.

remaining lobes, with the greatest relative increase in tissue volume and air volume occurring in the upper and cardiac lobes. Our results are consistent with the results of these studies, showing that the compensatory growth response after left pneumonectomy is not uniform among the lobes of the right lung, with the upper and cardiac lobes displaying an increased growth response compared with the middle and lower lobes.

One possible explanation for the higher volume increase in the cardiac lobe is that the anatomic location of this lobe might provide additional space for expansion than seen in the other 3 lobes. In fact, it has been reported in dogs that preventing lateral expansion of the remaining lung by an inflated prosthesis diminished approximately $70 \%$ to $80 \%$ the postpneumonectomy increase in lung volume. ${ }^{18}$ Other growth-promoting stimuli, such as the relative changes in lung ventilation and distension, might also contribute to the cardiac lobe growth response after pneumonectomy.

In the present study we examined the effect of left pneumonectomy on the vascular (arterial) growth of the right lung lobes. Arteriography showed that arterial area index increased in all lobes after pneumonectomy. Interestingly, the upper lobe showed a significantly greater increase in arterial area index when compared with the lower and cardiac lobes, values that correlate with increased lobe volume index, lobe weight index, and PCNA expression in the upper lobe. Although we did not observe pulmonary hypertension (as measured by right ventricular hypertrophy), we cannot rule out the possibility that chronic hypertension might develop after left pneumonectomy at a time beyond 21 days. We believe that this is unlikely, however, because we have previously reported a transient increase in pulmonary hypertension after left pneumonectomy in mice. ${ }^{19}$ In this study right ventricular pressure increased
$42 \%$ above baseline levels 1 day after pneumonectomy, which returned to baseline levels by day 3 and remained at baseline levels through day 14 . In addition, we have shown that 21 days is enough time for rats to experience right ventricular hypertrophy after 3 or 4 lobes of the right lung are removed. ${ }^{8}$

Because the upper lobe displayed the highest values in many of the growth parameters, we evaluated the length of 2 arterial segments in the upper lobe of rats in the pneumonectomy group compared with those in the sham group. We used the branch off of the right pulmonary artery to be representative of the vascular growth that was apparent in other areas and lobes of the lung. The length of branches was easily measured for this artery because it did not overlap with other arteries on the radiograph. We have used this approach previously to address the vascular response in the left lung after right lobectomy in rats. ${ }^{8} \mathrm{We}$ found that the length of both segments A and B significantly increased after pneumonectomy ( $59 \%$ and $33 \%$, respectively) compared with that in the sham group. Although these data refer only to the upper lobe, these findings demonstrate that increased arterial area index in postpneumonectomy lungs is not just due to increased perfusion or dilation of pre-existing arteries not normally visible but is an indication of true arterial growth. These results also support the relationship between arterial growth and increased compensatory growth.

Changes in relative distribution of pulmonary blood flow were assessed by using radiolabeled microspheres to address the question of whether an increase in blood flow might correlate with the differences in lobe growth encountered in our study. The right lungs of rats not undergoing operations showed the following blood-flow distribution: the upper lobe received the highest relative flow (33\%), 
A

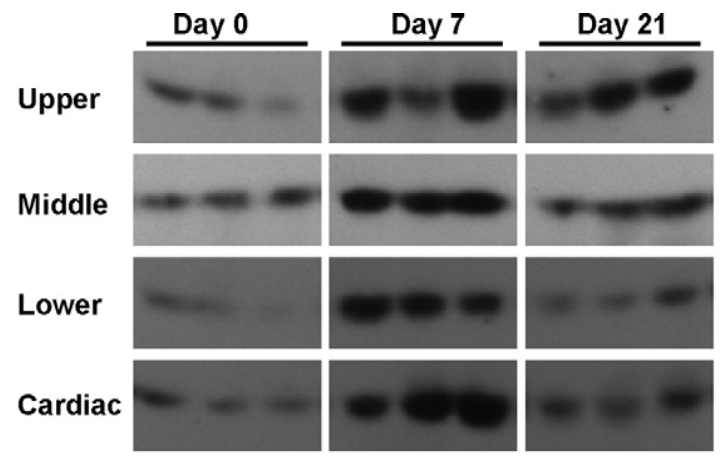

B
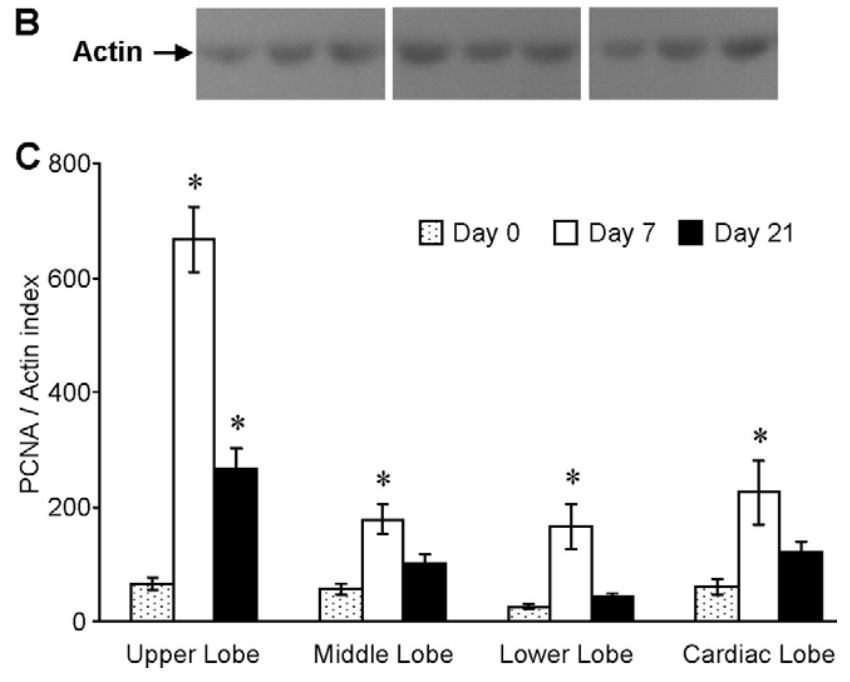

Figure 5. Changes in proliferating cell nuclear antigen (PCNA) expression in the right lung lobes after left pneumonectomy. A, Representative Western blots for proliferating cell nuclear antigen expression (36 kd) in each lobe at days 0, 7, and 21 after pneumonectomy. B, Western blot of actin (43 kd) expression in the middle lobe at 7 and 21 days after pneumonectomy versus that seen in animals not undergoing operations, documenting that surgical intervention did not alter actin expression. C, Histogram showing quantitation of proliferating cell nuclear antigen expression (as relative proliferating cell nuclear antigen/actin index) by means of Western blotting in each lobe at 7 and 21 days after pneumonectomy compared with corresponding lobes from animals not undergoing operations. Proliferating cell nuclear antigen expression was significantly increased in each lobe after pneumonectomy, with peak levels occurring at day 7 for each lobe ( ${ }^{*} \boldsymbol{P}<.05$ vs day 0 ). In addition, the upper lobe maintained a greater relative increase in proliferating cell nuclear antigen at day 21 ( ${ }^{*} P<.05$ vs day 0$)$.

followed by the cardiac (28\%), middle (26\%), and lower (13\%) lobes. After left pneumonectomy, a large increase in blood flow to the right lung occurs. However, the relative distribution of flow remained fairly constant in each lobe throughout the 21-day postpneumonectomy period. Postpneumonectomy blood blow distributions were similar to the values in rats not undergoing operations, where the upper lobe had a higher fraction of blood flow compared with the lower and cardiac lobes. We speculate that differential blood-flow distribution between the lobes can affect the growth response of those tissues. We found that higher blood flow (33\% in the upper lobe) was associated with higher vascular area index and proliferation rate, whereas the opposite occurred in the lower lobe, which received the smallest fraction of flow (13\%) and underwent the smallest growth response of all the lobes. An early study by McBride and associates ${ }^{20}$ suggested that increased pulmonary blood flow is likely not the predominant stimulus for compensatory lung growth. In this study the left caudal lobe pulmonary artery of ferrets was banded to prevent increased blood flow into that lobe after pneumonectomy. Despite no increase in flow after pneumonectomy, the caudal lobe still displayed significant, although somewhat reduced, compensatory growth. Therefore although vascular blood flow might not be the sole stimulus for compensatory growth, it might modulate the magnitude of the response, such that regions of the lung that receive a higher distribution of flow show a greater vascular growth response after pneumonectomy.

It has been well established that postpneumonectomy compensatory lung growth entails significant hyperplasia. We have previously shown that alveolar epithelial cell proliferation, by means of bromodeoxyuridine incorporation, peaks at 7 days after pneumonectomy. ${ }^{12}$ In addition, we have shown that the number of PCNA-positive cells is enhanced after pneumonectomy and that these cells largely include airway epithelial cells, alveolar type II cells, and endothelial cells of the pulmonary vessels. ${ }^{21}$ It should be noted that it is also possible that migratory cells of hemopoietic origin could also contribute to the increased PCNA levels observed in the lung by means of Western blot analysis, although this has not been specifically assessed. In the present study we have also presented evidence of hyperplasia, as indicated by increased PCNA expression, which peaks 7 days after pneumonectomy. Therefore we can speculate that the vascular growth we report in this study is part of a hyperplastic response in the remaining lung, potentially modulated by blood flow and other factors. Although not directly proved, increased vascular area, as well as segment lengths, are most likely indicators of arterial (hyperplastic) growth. One potential limitation of this study, however, is that recruitment of existing vessels that are not normally perfused under basal conditions could have contributed to the increase in vascular area that was observed after pneumonectomy. However, this is unlikely because the barium mixture was infused at high pressure (74 $\mathrm{mm} \mathrm{Hg}$ ), which should have perfused these vessels.

In summary, we demonstrated that left pneumonectomy induces compensatory growth in each of the 4 lobes of the 
right lung, including increased lobe weight and volume indices, arterial area index, and PCNA expression. These increases in growth and proliferation were not uniform among the lobes but were greater in the upper and cardiac lobes. We also showed a predominant vascular growth in the upper lobe characterized by an increase in arterial area index, prolonged upregulation of PCNA, and increased length of proximal arterial branches, all of which correlated with the highest fraction of relative blood flow in this lobe. Measurements of relative blood flow could not fully explain the disproportionate changes in the various parameters of lobar growth, particularly in the cardiac lobe, which is indicative of the complexity of compensatory lung growth responses. Other growth-promoting stimuli, such as the relative changes in lung ventilation and distension, might also contribute to the disproportionate lobar growth response after pneumonectomy.

We thank Kendra Hardy and Jeremy Gatesman at the University of Virginia Health System for their technical assistance with animal operations and microsphere injections and Patricia Pastura at Cincinnati Children's Hospital Medical Center for technical assistance with radiography.

\section{References}

1. Berger LC, Burri PH. Timing of the quantitative recovery in the regenerating rat lung. Am Rev Respir Dis. 1985;132:777-83.

2. Brody JS. Time course of and stimuli to compensatory growth of the lung after pneumonectomy. J Clin Invest. 1975;56:897-904.

3. Buhain WJ, Brody JS. Compensatory growth of the lung following pneumonectomy. J Appl Physiol. 1973;35:898-902.

4. Hsia CC, Herazo LF, Fryder-Doffey F, Weibel ER. Compensatory lung growth occurs in adult dogs after right pneumonectomy. $J$ Clin Invest. 1994;94:405-12.

5. Kuboi S, Mizuuchi A, Mizuuchi T, Taguchi T, Thurlbeck WM, Kida $\mathrm{K}$. DNA synthesis and related enzymes altered in compensatory lung growth in rats. Scand J Clin Lab Invest. 1992;52:707-15.

6. Rannels DE, White DM, Watkins CA. Rapidity of compensatory lung growth following pneumonectomy in adult rats. J Appl Physiol. 1979; 46:326-33.
7. Sekhon HS, Thurlbeck WM. A comparative study of postpneumonectomy compensatory lung response in growing male and female rats. J Appl Physiol. 1992;73:446-51.

8. Le Cras TD, Fernandez LG, Pastura PA, Laubach VE. Vascular growth and remodeling in compensatory lung growth following right lobectomy. J Appl Physiol. 2005;98:1140-8.

9. Gilbert KA, Rannels DE. From limbs to lungs: a new perspective on compensatory lung growth. News Physiol Sci. 1999;14:260-7.

10. Doerschuk CM, Sekhon HS. Pulmonary blood volume and edema in postpneumonectomy lung growth in rats. J Appl Physiol. 1990;69: 1178-82.

11. Landesberg LJ, Lee K, Rosengart TK, Crystal RG. Regulation of angiogenesis during post-pneumonectomy compensatory lung growth in rats [abstract]. Am J Respir Crit Care Med. 1999;159:A353.

12. Leuwerke SM, Kaza AK, Tribble CG, Kron IL, Laubach VE. Inhibition of compensatory lung growth in endothelial nitric oxide synthasedeficient mice. Am J Physiol Lung Cell Mol Physiol. 2002;282: L1272-8.

13. Kaza AK, Kron IL, Kern JA, Long SM, Fiser SM, Nguyen RP, et al. Retinoic acid enhances lung growth after pneumonectomy. Ann Thorac Surg. 2001;71:1645-50.

14. Roman CD, Hanley GA, Beauchamp RD. Operative technique for safe pulmonary lobectomy in Sprague-Dawley rats. Contemp Top Lab Anim Sci. 2002;41:28-30.

15. Scherle W. A simple method for volumetry of organs in quantitative steorology. Mikroskopie. 1970;26:57-60.

16. Haapasalo HK, Sallinen PK, Helen PT, Rantala IS, Helin HJ, Isola JJ. Comparison of three quantitation methods for PCNA immunostaining: applicability and relation to survival in 83 astrocytic neoplasms. J Pathol. 1993;171:207-14.

17. Ravikumar P, Yilmaz C, Dane DM, Johnson RL Jr, Estrera AS, Hsia CC. Regional lung growth following pneumonectomy assessed by computed tomography. J Appl Physiol. 2004;97:1567-74.

18. Hsia CC, Wu EY, Wagner E, Weibel ER. Preventing mediastinal shif after pneumonectomy impairs regenerative alveolar tissue growth. Am J Physiol Lung Cell Mol Physiol. 2001;281:L1279-87.

19. Maxey TS, Fernandez LG, Reece TB, Keeling WB, Kron IL, Laubach VE. Endothelial nitric oxide synthase is essential for postpneumonectomy compensatory vasodilation. Ann Thorac Surg. 2006;81:1234-8.

20. McBride JT, Kirchner KK, Russ G, Finkelstein J. Role of pulmonary blood flow in postpneumonectomy lung growth. J Appl Physiol. 1992; 73:2448-51.

21. Li D, Fernandez LG, Dodd-o J, Langer J, Wang D, Laubach VE. Upregulation of hypoxia-induced mitogenic factor in compensatory lung growth after pneumonectomy. Am J Respir Cell Mol Biol. 2005 $32: 185-91$ 